\title{
Types définis et types intermédiaires dans la mitose des végétaux
}

\author{
par \\ André Eichhorn, Paris
}

Reçt le 2 septembre 1933

\section{Introduction}

Jusqu'en ces dernières années tous les cytologistes professaient une même opinion tant sur la structure du noyau quiescent que sur le mode de formation des chromosomes au début de la cinèse et sur l'évolution de la cinèse elle-même. D'après cette manière de voir, unanimement admise, on considérait le noyau comme formé par un réseau de chromatine destiné, lors de la reprise de l'activité mitotique, à donner les chromosomes, par une sorte de découpage orienté. En dehors de ce réseau on indiquait la présence de plusieurs nucléoles, tous ces éléments baignant dans un suc nucléaire que délimite une mince membrane.

Les divergences d'opinions ne portaient guère que sur la façon d'envisager la reconstitution du réseau, sur le mode d'aggrégation de ce dernier et, en troisième lieu, sur les relations entre nucléoles et chromosomes. Pour certains, le reticulum se formerait par suite du clivage longitudinal des chromosomes, ce qui aurait pour résultat de les partager et de les réduire à l'état de fins filaments qui s'enchevêtreraient ensuite en un réseau à mailles serrées. D'après d'autres earyologistes, les chromosomes seraient attaqués par le dehors et subiraient ainsi un amincissement progressif qui conduirait à la reconstitution du reticulum. Quant à la structure proprement dite de celui-ci, deux opinions prévalaient: les uns admettaient qu'il offrait un aspect granuleux; les autres qu'il était formé par des filaments excessivement minces. En ce qui concerne, enfin, les rapports entre nucléoles et chromosomes, on pensait, le plus souvent, que ceux-ci empruntaient à ceux-là une partie de leur substance pour achever, si l'on peut s'exprimer ainsi, leur édification.

De la sorte on put adopter un commun schéma de la mitose valable pour tous les végétaux et ne comportant que quelques variantes sur les points de détail litigieux qui viennent d'être indiqués. C'est à STrasBURGER que revient le mérite d'avoir fait connaitre ce schéma sur 
lequel on a, depuis, pris modéle dans toute description de mitoses chez les végétaux. Que cela ait été possible s'explique aisément si l'on considère, d'une part que, d'une façon générale, les caryologistes se sont, pendant longtemps, adressés sans cesse aux mêmes objets et spécialement à des objets possédant des chromosomes de grande taille et, d'autre part, ont utilisé constamment les mêmes méthodes de fixation sans en faire une étude critique ni contrôler les résultats obtenus par des observations in vivo.

Assez récemment seulement les progrès réalisés en cytologie ont permis aux caryologistes de faire la critique des méthodes en usage jusqu'ici et d'avoir davantage recours à l'observation vitale, ce qui eut pour effet, en leur permettant d'étendre leurs conclusions, de les inciter à étudier le plus grand nombre possible d'exemples.

J'ai montré ailleurs la nécessité qu'il y avait de s'assurer de bonnes fixations contrôlées et je n'y reviendrai pas. Je voudrais simplement montrer pourquoi, à l'heure actuelle, un unique schèma ne peut plus suffire pour la description de la mitose somatique chez les végétaux et exposer les résultats auxquels l'examen, tant in vivo qu'après fixation, d'un matériel varié, m'a conduit.

\section{Les deux types définis de noyaux quiescents}

1) NoYAUX PRÉSEnTANT UN RÉSEAU SIMPLE.-Il n'est rien de si aisé que de vérifier la présence, dans de très nombreux noyaux, d'un réticulum-décrit depuis longtemps d'ailleurs-et ceci quelle que soit la méthode employée. Ce réseau, signalé autrefois par STRAsBURGER et ses élèves, et que l'on trouve indiqué dans la majorité des travaux publiés depuis lors et les ouvrages généraux (TISCHLER, SHARP), apparaît avec une aussi parfaite netteté sur le vivant que sur les préparations fixées. Si son existence ne peut plus, semble-t-il, prêter à discussion maintenant, sa structure n'est pas interprêtée par tous de la même façon.

A la suite d'observations vitales sur l'Allium, l'Hyacinthus, le Triticum, etc., il me paraît être constitué par un ensemble de très fins granules plutôt que par de véritables filaments. Autrement dit, je ne crois pas que le réseau résulte de l'enchevêtrement de fins trabécules qui se recouperaient les uns les autres en certains points où ils offriraient l'apparence de noeuds. En effet, au cours des observations vitales que j'ai faites, je n'ai pas rencontrè de ces sortes de renflements qui se coloreraient après fixation d'une façon plus intense et ceci en rapport 
avec leur masse. Au contraire, le réseau m'est toujours apparu comme régulier et sans aucun granule plus épais que les voisins. A mon avis l'apparence filamenteuse du réseau, constatée et indiquée très souvent à la suite d'observations sur coupes fixées et colorées, s'explique par l'action du fixateur qui soude les uns aux autres tous les granules de chromatine en lesquels les chromosomes ont été comme pulvérisés au moment de la télophase précédant le passage à l'état de repos, durable ou momentané.

Par contre, cet aspect filamenteux correspond à la réalité lorsqu'il est observé au moment de la reprise de l'activité nucléaire et l'état filamenteux peut être considéré comme opérant la transition entre le stade de repos proprement dit, où l'apparence du noyau est nettement granuleuse, et celui de début de la prophase où les chromosomes se dessinent sous la forme de longs filaments grêles et sinueux. On voit fort bien, sur le vivant, s'opérer le passage d'une phase à l'autre.

2) NOYAU SANS AUCUN RÉSEAU.-Ainsi qu'il l'a été rappelé plus haut, dans le schéma général de la mitose, tel qu'il avait été adopté par les cytologistes végétaux, le point de départ était invariablement le réseau de chromatine qui, par fragmentation, fournissait les chromosomes. Il n'avait jamais été admis par quiconque qu'il puisse, chez certaines plantes, n'en pas exister.

Ceci s'explique très facilement si l'on tient compte de différentes choses. Tout d'abord les caryologistes s'adressaient normalement à des objets où la présence d'un réseau est constante. De fait leur attention était attirée plus particulièrement sur les chromosomes de longue taille qui dérivent, comme nécessairement, d'un réseau. D'autre part, les fixateurs utilisés par la plupart, sinon la totalité, des caryologistesmême encore à l'heure actuelle-étaient surtout à base d'acide acétique. Or, ces liquides ont la propriété de coaguler le suc nucléaire lui-même de telle sorte qu'ils déterminent des apparences de réseau dans des noyaux qui en sont certainement dépourvus. Faut-il ajouter que, le plus souvent, les observations des cytologistes portaient sur des stades dèjà avancés de la cinèse et que rares étaient ceux qui se préoccupaient esentiellement de la structure du noyau quiescent, par exemple. Enfin jamais dans les études poursuivies sur le vivant, par les anciens auteurs, il n'était tenu compte de la vitalité de la cellule que l'on ne pensait pas et que, d'ailleurs, on savait mal contrôler faute de critérium convenable.

On saisit de la façon la plus évidente de quelle importance a été le perfectionnement de la technique pour la compréhension des phénomènes nucléaires, car ce n'est que grâce à l'usage de fixateurs respec- 
tant parfaitement l'économie nucléaire et au contrôle des résultats, rendu possible par l'observation vitale, que j'ai pu affirmer que, dans certains exemples, le noyau ne possédait pas une structure réticulée au repos.

L'étude des cellules animales avait, du reste, déjà conduit certains cytologistes à admettre l'absence d'un réseau dans de nombreux cas, mais cette conception n'avait pas trouvé de partisans bien fermes chez les botanistes, de telle sorte que l'existence de noyaux " homogènes" n'était pas admise par eux (TISCHLER) et, cependant, de tels noyaux ne sont pas rares chez les végétaux. Toutefois, il convient de faire remarquer que l'expression, utilisée en caryologie animale, de " noyaux homogènes" n'est pas absolument correcte, ainsi que la suite de cet exposé en témoignera.

En effet. l'observation in vivo de noyaux dans les méristèmes radiculaires de végétaux comme les Cucurbita, les Thladiantha, le Bolbostemma ou les Sinapis permet de constater qu'ils sont essentiellement constitués par un enchylème nucléaire délimité par une membrane très nette, un nucléole relativement volumineux, sphérique, unique et généralement situé au centre du noyau. Outre ce nucléole, qui retient évidemment de prime abord l'attention, on note la présence de corpuscules arrondis ou ovoïdes, plus ou moins aplatis le long de la membrane et que, en raison de leur taille réduite, et de leur faible réfringence, on pourrait ne pas reconnaître à une observation superficielle. Pour faire cette étude in vivo il faut s'assurer de la vitalité de la cellule et prendre soin, évidemment, de ne pas prolonger trop longtemps l'examen.

On voit, par tout ce qui précède, que l'appellation de " noyaux homogènes" pour désigner ceux de ces corps qui ne possèdent pas un réseau de chromatine n'est pas correcte puisqu'aussi bien on y peut distinguer une structure d'un type particulier. II me paraît logique de les désigner sous le nom de "noyaux à prochromosomes" et voici pourquoi.

Tout d'abord, dans le cas des noyaux de ce type, la chromatine est localisée exclusivement sur les élèments que l'on trouve à la périphérie du noyau, d'autre part, ces corps évoluent directement, au moment de la reprise de l'activité nucléaire, en chromosomes proprement dits. Enfin, nous verrons tout à l'heure que leur devenir en télophase justifie de diverses manières cette dénomination.

En résumé, on doit admettre qu'il existe deux types bien définis de structure du noyau végétal quiescent: l'un caractérisé par la présence d'un reticulum constitué par de multiples et fins granules de chromatine; l'autre caractérisé inversement par l'absence de tout réseau et 
chez lequel la chromatine se trouve localisée sur un nombre restreint de gros granules situés normalement à la périphérie du noyau, tout contre la membrane et qui sont de véritables " prochromosomes". L'expression souvent utilisée de " noyaux homogènes " ne convient évidemment en aucune manière pour désigner des noyaux de ce dernier type, on peut même ajouter que cette dénomination n'est pas heureuse dans le cas des cellules animales puisque, si l'enchylème ne supporte apparemment, in vivo, aucun réseau de chromatine, on distingue toujours, parfaitement, un ou plusieurs nucléoles dans la cavité nucléaire dont l'apparence n'est, partant, pas “ homogène".

\section{Les deux types intermédiaires de noyaux quiescents}

1) Noyaux RÉTICULÉs A CHROMOCEnTREs. - Les deux types de structure nucléaire qui viennent d'être étudiés sont facilement opposables l'un à l'autre. Il aurait été intéressant de pouvoir les attribuer respectivement à des groupes végétaux bien définis, mais, on l'a vu, on rencontre chez les Angiospermes tantôt l'un tantôt l'autre, et parfois à l'intérieur d'une même famille ils comptent tous deux des représentants, c'est le cas chez les Cucurbitacées, par exemple. Par contre, on trouve, chez les Conifères, une réelle uniformité de structure nucléaire à laquelle nous avons donné le nom de type $P$ inus ou à chromocentres, cette uniformité est telle que, à première vue, il est possible en considérant une préparation, de la reconnaître comme se rapportant à un représentant de ce groupe, et par contre qu'il est impossible de différencier l'une de l'autre deux préparations obtenues à partir de deux Conifères différentes tant elles sont identiques en tous points : stade de repos ou évolution de la mitose, forme ou taille des chromosomes.

Dans un noyau au repos d'une Conifère on rencontre exactement le même réseau, constitué par un ensemble de fins granules, qui a été décrit plus haut chez certaines Angiospermes. Mais on trouve, en outre, en assez grand nombre, de gros granules mélangés aux plus fins. Nous avons réservé à ces granulations de chromatine le nom de chromocentres en raison de leur évolution lors de la mitose sur laquelle nous reviendrons plus loin. Dans ce cas non plus la structure ne saurait être considérée comme filamenteuse, elle ne le devient, de la même maniére que chez les Angiospermes étudiées précédemment, qu'au moment de l'entrée du noyau en cinèse ainsi que l'indiquent de la façon la plus nette les préparations d'objets fixés.

On voit que chez les Conifères on a affaire à une structure nucléaire 
d'un type un peu différent de celui des Angiospermes offrant un réseauet qui, lui, est parfaitement défini-et qu'elle constitue vraiment un type intermédiaire, d'autant plus que le nombre des granulations chromocentriques paraît absolument variable ou en tout cas ne saurait être précisé.

2) NOYAUX NON RÉTICULÉs À CHROMOCENTRES. - Il existe un quatrieme type de structure nucléaire pour lequel nous proposons le nom de type: Fatshedera qui n'a jamais été signalé et qui, cependant, paraît être assez répandu puisque nous l'avons rencontré fortuitement chez des végétaux aussi différents que Gossypium, Fatshedera et qu'il vient d'être signalé par R. NARDI chez les Hépatiques (Lunularia). Dans ces divers cas, qui ne sont d'ailleurs pas isolés et auxquels d'autres viendront certainement s'ajouter comme permettent de le penser des études en cours, le noyau au repos, in vivo comme après fixation, ne laisse déceler à son intérieur aucun réseau de granules de chromatine, mais en dehors du nucléole volumineux et généralement unique, on trouve, dans la cavité nucléaire, une assez grande quantité de gros grains de chromatine dispersés et à quelque distance les uns des autres, sans liaison entre eux. Ils rappellent si l'on veut, mais en plus petit, les prochromosomes avec cette différence, toutefois, que tout d'abord ils sont plus petits et, en second lieu, qu'ils ne sont pas localisés presque exclusivement à la périphérie du noyau, mais dispersés dans toute la cavité nucléaire. Ce sont eux qui en s'unissant par deux ou trois constituent les chromosomes de la prophase. Il ne semble pas utile de créer un terme nouveau pour désigner ces granules d'un genre sans doute spécial, mais en tout comparables aux chromocentres, à cette exception près qu'ils ne sont pas mélangés à d'autres granules plus petits ainsi que cela se produit chez les Conifères. Leur évolution, que nous envisagerons plus loin, permet en tout état de cause cette assimilation avec, cela va sans dire, quelques restrictions sur lesquelles nous aurons l'occasion d'insister.

Cette fois encore, comme dans le cas des Conifères, le type de structure est nettement intermédiaire. Il l'est de la façon la plus évidente entre le type Pinus, par suite de l'absence d'un réseau, et celui des noyaux à prochromosomes dont il diffère par le nombre des grains de chromatine et leur devenir un peu plus compliqué.

De tout ce qui précède on peut, me semble-t-il, conclure à l'existence de quatre types principaux de structure nucléaire, tout noyau répondant à l'un quelconque d'entre eux. Nous montrerons tout à l'heure comment évoluent les noyaux de ces diverses sortes et de quelle manière se forment les chromosomes dans l'un et l'autre cas, nous verrons alors 
qu'il est possible de ramener toute évolution nucléaire à un des quatre schémas et que de plus il est difficile, même hypothétiquement, d'envisager l'existence tant de modes de division autres que de types de structure différents.

\section{La cinèse dans les quatre types de noyaux}

Nous ne considérerons, dans les pages suivantes, que les deux stades de la mitose vraiment critiques : la prophase et la télophase, car c'est à la première que se dessinent les chromosomes et il est intéressant de saisir, dans le détail, leur réapparition et, d'autre part, c'est à la seconde que s'estompent, en quelque sorte, ces mêmes chromosomes et que l'on assiste à la réédification des noyaux au repos sur lesquels nous venons d'insister un peu longuement. Nous ferons une exception pour les Conifères pour lesquelles nous donnerons une brève étude de la métaphase, car c'est à ce stade que se produit le clivage chromosomique chez ces végétaux, ce qui constitue un caractère assez particulier et qui peut servir à leur différenciation caryologique.

\section{La prophase}

1) LES NOYAUX $\dot{A}$ RÉSEAU SIMPLE.-L'évolution des noyaux de ce genre répond très exactement au schéma général que l'on est habitué de donner pour la mitose chez les végétaux. Les exemples susceptibles de l'illustrer sont nombreux et peuvent être empruntés aux Cryptogames vasculaires ainsi qu'il résulte des travaux de $R$. de LITARDIÈRE sur les Filicinées, aux Angiospermes, c'est le cas de Vicia, Allium, Hyacinthus, Cyclanthera, etc., ou encore aux Gymnospermes et c'est parmi celles-ci le Ginkgo biloba qui paraît seul s'y rapporter.

Nous avons indiqué précédemment que dans le noyau au repos la chromatine se trouvait dispersée sous forme de petits granules dont l'ensemble constitue un fin reticulum granuleux. Au moment de l' entrée du noyau en activité, ces fins granules paraissent s'accoler les uns aux autres suivant une disposition et d'après des lois qui nous échappent et réaliser alors un réseau d'apparence filamenteuse. Ceci se marque surtout nettement sur les préparations d'objets fixés, mais cette évolution peut pareillement être suivie in vivo et l'on voit alors la stru ture du noyau devenir plus apparente. Cette image ne tarde pas à s'accentuer davantage et les filaments constituant, à ce moment, le réseau semblent prendre une certaine orientation qui se reconnait naturellement encore mieux sur préparations. 
Ils apparaissent alors tendus d'un côté à l'autre du noyau et plus ou moins spiralés; ils rappellent, si l'on veut, une sorte de ressort légérement distendu. Il ne semble plus qu'il y ait de points de contact entre eux, à en juger du moins par ce que l'on distingue sur le vivant ou dans de bonnes préparations, car après action de liquides fixateurs que l'on doit considérer comme nocifs l'ensemble des filaments offre l'apparence d'un peloton et peut parfois donner l'impression qu'il existe un spirème, c'est là une des nombreuses erreurs d'interprétation dues à l'usage de mauvais fixateurs et dont il faut se garder. Un peu plus tard ces filaments se raccourcissent sensiblement et l'on peut, dans certains cas, voir de la façon la plus nette les deux extrémités de chacun d'eux. On est fondé dès lors à les désigner déjà sous le nom de chromosomes proprement dits, puisqu'ils ne subiront plus de bien grands changements avant de présenter leur aspect définitif. Cette transformation consiste surtout en un tassement sur eux-mêmes, ce qui entraîne leur épaississement. A un stade plus ou moins précoce, soit alors qu'il a encore l'aspect de spires, soit seulement après son raccourcissement, il apparaît une fente longitudinale tout le long de l'élément chromosomique ; cette fente s'estompe ultérieurement pendant un peu de temps, cependant que le filament s'épaissit davantage et diminue de longueur pour autant.

Il convient d'insister ici sur un des stades primordiaux de la prophase auquel on peut donner le nom de stade des "filaments minces".

Ce stade prophasique de filaments minces fissurés est, en effet, absolument particulier à ce type de structure nucléaire et à ce mode de mitose, nous ne le rencontrerons plus dans les descriptions ultérieures. Il se retrouve d'ailleurs, dans les exemples que nous examinons maintenant avec une parfaite régularité et chez toutes les espèces qui le présentent les chromosomes se ressemblent plus ou moins et parfois étonnamment à ce moment de la cinèse. Ce n'est qu'un peu plus tard, quand le clivage s'est à nouveau estompé et que le chromosome s'est raccourci, que l'aspect de chacun d'eux est caractéristique dans chaque espèce.

2) ChEZ LES VÉGÉtAuX À PROCHRomosomes. - Rien ne marque dans ce cas le passage de l'état quiescent à l'état d'activité. On ne peut même pas dire qu'au moment de la rentrée du noyau en division les prochromosomes manifestent une chromaticité plus grande car sur des éléments aussi petits il est difficile de constater des différences de ce genre et on sait, de plus, le peu de valeur qu'aurait un tel critérium éminemment subjectif et à la merci d'une différenciation plus 
ou moins poussée. Morphologiquement, on ne peut, non plus, reconnaître de modifications typiques des prochromosomes. Ce n'est donc que lorsqu'on les voit quitter la membrane nucléaire qu'il est possible de se rendre compte du début de la mitose.

Progressivement, mais toutefois assez vite, les prochromosomes se dirigent vers le centre du noyau et se rassemblent autour du nucléole. Chez ces exemples, Cucurbitacées, Sinapis, etc., tout est simplifié au maximum. Le chromosome ne subit pas de ces transformations nombreuses, propres aux chromosomes de longue taille. Il est immédiatement constitué. Parfois il s'étire légèrement pour prendre une forme en bâtonnet. Nous avons signalé sa persistance durant l'état quiescent, sous forme de prochromosome; étant donné que nous avons affaire là à des chromosomes très petits, il n'y a rien d'étonnant à ce que les modifications subies soient de faible amplitude.

Les prochromosomes, dont j'ai contribué à démontrer l'existence et dont j'ai fait ensuite une particulière étude, avaient déjà été entrevus par LUNDEGÅRDH chez Cucurbita dès 1910, mais par suite d'une technique insuffisante il n'avait saisi ni l'importance de ce qu'il dénommait: les caryosomes, ni leur nature véritable et moins encore avait-il compris leur évolution.

C'est à $R$. de LITARDIÈre que l'on doit la première description précise d'une mitose à prochromosomes. Il a, en effet, rencontré, en étudiant la caryologie des Filicinées, une mitose d'un genre particulier chez Azolla, au sujet de laquelle il s'exprime ainsi: "Chromosomes petits se présentant sous une forme sphérique ou ovalaire dans les noyaux interphasiques, mais subissant un léger allongement au cours de l'anachromase; pas d'alvéolisation à la catachromase."

Il semble bien que les phénomènes décrits chez Azolla correspondent parfaitement à ceux que j'ai fait connaitre plus tard chez les Cucurbitacées entre autres. Mais de LITARDIÈRE n'a pas songé, quand il écrivait ces lignes, que ce type de mitose puisse être aussi général et se retrouver chez un certain nombre de végétaux. De même ScHILleR lorqu'il décrivit la caryocinèse du Capparis spinosa, plante à prochromosomes, n'a pas soupconné qu'il pouvait y avoir de nombreux exemples du même genre. On peut dire, en effet, que les Dauerchromosomen figurés par cet auteur correspondent parfaitement aux prochromosomes tels que je les ai définis. Aucun de ces auteurs ne paraît donc avoir tiré de ses recherches les conclusions qui en découlent naturellement. Il ne semblera, toutefois, pas téméraire, pensons-nous, de donner comme type défini cette mitose d'un genre encore peu étudié mais qui compte- 
rait, à en juger par des examens actuellement en cours chez diverses Angiospermes, d'autres représentants.

3) LES VÉGÉTAUX DONT LES NOYAUX COMPORTENT UN RÉSEAU ET DES CHROMOCENTRES. - Bien que dans ses grands traits la prophase chez les Conifères paraisse identique à celle des Angiospermes à longs chromosomes, elle en diffère profondément dans le détail, comme on va en pouvoir juger.

Dans le noyau au repos on trouve, ainsi qu'il l'a été indiqué plus haut, un réseau de chromatine finement granuleux agrémenté d'un assez grand nombre de grumeaux suffisamment gros auxquels on a donnè le nom de chromocentres. Lorsque le noyau va entrer en mitose les granules, petits et gros, s'unissent pour constituer des filaments renfiés aux endroits où se rencontrent les chromocentres. L'aspect de ces filaments est dès l'abord beaucoup plus grossier que dans l'autre cas, de plus on ne rencontre jamais d'images que l'on puisse rapporter au stade des " filaments minces" que nous avons reconnu chez d'autres exemples. En effet, au cours de la prophase, aucune fissuration n'apparaît à l'intérieur des chromosomes chez les Conifères, ce qui constitue vraiment un caractère distinctif surtout pour des espèces à chromosomes spécialement longs. Outre que l'élément chromosomique est dès sa reconstitution relativement épais, sa disposition à l'intérieur de la cavité nucléaire n'est pas la même que dans le cas des Angiospermes à chromosomes allongés, étudiées précédemment. Il est beaucoup plus rigide et ne présente pas cet aspect spiralé que nous avons reconnu aux autres chromosomes longs et cela s'explique ici tout naturellement par son épaisseur relative.

Il ne conserve pas longtemps d'ailleurs cette forme allongée et son tassement est assez rapide. Au cours de ce processus, les nodules placés de ci de là le long du chromosome servent, en quelque sorte, de centres de condensation de la chromatine, en ce sens que personnellement ils ne subissent pas de modifications sensibles mais que, par contre, les portions amincies situées entre eux se tassent et s'épaississent, dans une proportion directe, pour amener à une épaisseur uniforme tout le corps chromosomique dont les contours finissent par se régulariser, ceci, du reste, très vite.

On voit combien cette évolution diffère, pour chacune des phases, de celle examinée précédemment, cette dernière étant connue depuis longtemps déjà et servant toujours de modèle aux descriptions des auteurs.

La métaphase chez les Conifères. - Nous nous occupons de ce stade ici parce que c'est à ce moment que la division des chromosomes s'opère 
chez les Conifères. Sans qu'il y ait jamais eu de fissuration prophasique, et aprés que les chromosomes se sont installés très régulièrement en plaque équatoriale, et alors seulement, il apparaît une fente longitudinale très nette dans tous les chromosomes simultanément, du moins à en juger par les images que nous eûmes sous les yeux, et où, en règle générale, tous les chromosomes étaient uniformément fissurés. On peut considérer cette division, définitive du premier coup, et non précédée, comme c'est le cas général, d'une préfissuration prophasique, comme un nouveau caractère distinctif de la mitose des Conifères, qui, ajouté à tous ceux que nous avons signalés déjà, et à ceux également typiques de la télophase que nous verrons plus loin, donne, à cette cinèse, une apparence toute particulière.

La description de la caryocinèse chez les Conifères, sujet qui n'avait jamais été abordé jusqu'ici, nous permet de bien saisir la distinction qu'il y a lieu de faire entre prochromosomes et chromocentres, distinction sur laquelle j'ai suffisamment insisté ailleurs et ne reviens que pour rappeler les définitions que j'ai pensé pouvoir proposer des deux termes. On désigne, en premier lieu, sous le nom de prochromosomes les granulations fortement chromatiques que l'on peut voir placées à la périphérie du noyau quiescent, contre la membrane, et qui ne sont autre chose que de véritables chromosomes ayant persisté sans s'être alvéolisés au cours d'une précédente mitose. D'autre part, suivant l'expression de BACCARINI, on appelle du nom de chromocentres les granules chromatiques épars sur le réticulum, tels qu'on les rencontre chez les Conifères spécialement. Il ne s'agit, cette fois, que de fragments de chromosomes qui, au cours de la prophase, s'intégrent dans les chromosomes nouvellement formés, après avoir joué le rôle de centres de condensation de la chromatine.

Ces définitions étant données, ce serait peut-être le lieu de discuter certaines idées récemment formulées au sujet de la reconstitution des chromosomes à la prophase par HeITz et par KuHN, mais il me paraît que cette discussion sera mieux à sa place à la suite de l'exposé qu'il nous reste à faire d'un dernier type de prophase, dont nous sommes encore en train de poursuivre l'étude et que l'on rencontre chez les noyaux non réticulés à chromocentres.

4) LES VÉGÉTAUX DONT LES NOYAUX NE COMPORTENT PAS DE RÉSEAU MAIS UNIQUEMENT DES CHROMOCENTRES.-Nous avons considéré la structure des noyaux quiescents, répondant à ce type (Fatshedera, Gossypium, Lunularia), comme intermédiaire; à juste titre on peut qualifier de la même façon la prophase chez ces 
végétaux. Les chromocentres, nous l'avons vu, sont assez nombreux dans la cavité nucléaire et ne sont pas, comme c'est le cas pour les prochromosomes, de préférence situés à la périphérie, contre la membrane, mais épars. Ils ne sont reliés entre eux par aucun tractus visible. Au début de la prophase plusieurs chromocentres voisins, deux ou trois, le plus souvent, se rejoignent et s'accolent, puis fusionnent intimement. Le chromosome est formé. En règle générale cet élément d'abord d'aspect irrégulier prend une forme plutôt allongée car il s'amincit et s'incurve légèrement. Sa taille est intermédiaire entre celle, des prochromosomes et celle des chromosomes dits longs, tels ceux de Vicia ou de Pinus, par exemple. Toutefois elle se rapproche plus sensiblement de celle du premier élément que de celle des seconds. Cette fois encore, nous notons l'absence du stade dit des "filaments minces ", stade qui paraît donc être caractéristique des Angiospermes à chromosomes longs. D'autre part, il s'opère, après la reconstitution du chromosome, une lègère fissuration, fugitive d'ailleurs, et qui réapparaît naturellement à la métaphase, moment où les chromosomes se clivent.

Nous avons employé dans la description de cette mitose le nom de chromocentres car il nous a paru inutile de créer, pour désigner ces éléments, un nom nouveau. En effet, si l'on se reporte à la définition que nous avons donnée plus haut de ce terme, on voit qu'elle convient parfaitement dans le cas présent. On a bien affaire, en fait, à des fragments de chromosomes ayant persisté au cours de la télophase précédente et qui confluent pour reformer de nouveaux chromosomes.

Voyons, maintenant que nous avons donné une description assez complète des divers types de structure des noyaux au repos et des différents modes de formation des chromosomes à la prophase, quelles sont les idées récentes émises sur ce sujet.

Deux auteurs modernes ont admis l'existence de chromocentres chez les végétaux et les considérent comme des fragments de chromosomes conservés intacts au delà de la télophase, dans le noyau au repos. Leurs interprétations diffèrent surtout au sujet du devenir de ces formations. Pour KuHN, elles ne persisteraient que pendant un temps et finiraient par disparaitre. De toute façon, elles n'existeraient plus au début de la prophase et les chromosomes se reformeraient aux dépens de la substance achromatique éparse dans la cavité nucléaire. Pour HEITZ, les chromocentres constitueraient des portions spéciales du chromosome qui seules demeureraient visibles lors du passage à l'état quiescent ou de repos et les chromosomes se reformeraient aux dépens 
de ces chromocentres et d'une substance achromatique qui se joindrait à ces chromocentres autour desquels elle se regrouperait.

Sans doute les chromocentres décrits par ces deux caryologistes sont-ils de véritables chromocentres. Mais je ne crois pas que la description qu'ils donnent l'un et l'autre de leur évolution soit exacte. Et, tout d'abord, en ce qui concerne l'opinion de KUHN, il ne me paraît pas qu'il puisse être donné le nom de chromocentres à des formations destinées à s'estomper et qui ne doivent plus, théoriquement, servir à rien. Mais ceci n'est que d'importance secondaire. Quant à leur disparition au début de la prophase, jamais je n'ai assisté à rien de pareil dans aucun des cas des objets soit à prochromosomes, soit à chromocentres que j'ai étudié. Bien au contraire, ces éléments persistent dans les noyaux des tissus adultes. D'autre part, jamais je n'ai vu chez aucun végétal réapparaître les chromosomes soudain au sein d'une masse amorphe, sous la forme d'un fin filament destiné à grossir ultérieurement. Toujours les chromosomes m'ont paru se reconstituer à partir de formations définies et très nettes. Toutes les idées de KUHN me semblent donc contraires à celles que j'ai pu acquérir au cours de mes observations.

Quant aux opinions de HEITZ, elles me paraissent davantage correspondre aux conclusions auxquelles je suis personnellement parvenu. Je ne crois pas, toutefois, que le réseau puisse jamais être parfaitement achromatique et que, dans certains cas, les chromocentres seuls soient visibles. Quant à moi je n'ai jamais vu réapparaître, à la prophase, une portion de chromosome en relation avec un ou plusieurs chromocentres qui eux n'auraient jamais cessé d'être distincts. Je crois que les observations de ces deux auteurs doivent s'interprêter de la façon suivante. Tout d'abord, les cas signalés par eux de réseau entièrement ou partiellement achromatique et aussi de noyaux homogènes peuvent être dus à une régression trop poussée qui ne permet plus de reconnaître la présence du réseau de chromatine, mais qui, dans certains cas, conserve encore les chromocentres. Ensuite, nous ne nous étonnons pas que l'on décrive des noyaux chez lesquels on ne trouve aucune formation autre que les chromocentres. C'est ce que nous avons vu plus haut chez le Fatshedera, entre autres. Je crois que dans les cas relatés par HEITz, par exemple, et où il existe de nombreux chromocentres, ceux-ci fusionnent ainsi que je l'ai indiqué précédemment pour donner les chromosomes à la prophase. Il pourrait se faire aussi, que dans plusieurs cas les auteurs aient décrit, comme chromocentres, de simples prochromosomes et l'on est, en effet, un peu étonné lorsque 
l'on confronte les conclusions de Schiller et de KuHN au sujet de Capparis. L'un étant assuré de se trouver en présence de chromosomes pérennants, le second estimant qu'il devait avoir affaire à des chromocentres dont on s'explique mal, au surplus, le devenir si l'on se réfère soit au texte, soit aux figures.

\section{La Télophase}

Nous n'envisagerons pas, pour y étudier les phénomènes télophasiques, les divers types de noyaux dans l'ordre adopté pour l'examen de la prophase. Sans doute est-on accoutumé de dire que ces deux phases sont inverses et comportent les mêmes processus qui simplement se déroulent en marche contraire, et ceci est parfaitement exact en ce sens que, si la prophase est un regroupement, une synthèse, la télophase est une dispersion, une analyse. Je voudrais souligner ici l'apparente désagrégation que subissent les chromosomes en télophasedésagrégation nulle dans les noyaux à prochromosomes, désagrégation apparemment maximale chez des Angiospermes à réseau simple.

$\left.1^{\circ}\right)$ Type Cucurbita. - A la prophase, les chromosomes quittent, nous l'avons vu, la membrane nucléaire et se groupent au centre du noyau et évoluent ensuite tels quels ou après s'être lègèrement ètirés comme chromosomes proprement dits. Inversement, en télophase, ces mêmes chromosomes qui, naturellement, se sont clivés et ont récupéré ensuite leur taille normale, se dirigent vers la périphérie du noyau et passent ainsi à l'état de prochromosomes dans le noyau quiescent. Cette évolution est la plus simple que l'on puisse concevoir et elle ne comporte aucune modification soit de la forme, soit de la constitution du chromosome qui n'est jamais attaqué comme le sont ceux des noyaux d'autres types.

$2^{\circ}$ ) Type Fatshedera. - A la télophase, les chromosomes subissent une sorte de désagrégation qui les transforme en un certain nombre de chromocentres plus petits, destinés à persister sous cette forme dans le noyau au repos après s'être dispersés dans la cavité nucléaire. Ici, par conséquent, les chromosomes se scindent en plusieurs nodules assez volumineux et la fragmentation dont ils sont l'objet est peu poussée, aussi bien est-ce le mode le plus simple de transformation. Il n'est réalisé que dans le cas de chromosomes de taille relativement petite. Les phénomènes sont plus compliqués, somme toute, la fragmentation est plus poussée quand il s'agit de chromosomes longs. Deux cas sont alors à envisager, ce sont, par 
ordre de complication des processus, celui des noyaux du type Pinus et ensuite celui des noyaux du type Allium.

$3^{\circ}$ ) Type PinUs. - Avant de traiter ce sujet, il convient de souligner un fait important, savoir que dans les deux exemples qu'il nous reste à examiner, le chromosome n'est jamais attaqué, si l'on peut s'exprimer ainsi, par le dedans. Autrement dit, il ne se produit pas, à la télophase, de scission longitudinale du chromosome, capable de rappeler les boutonnières caractéristiques du clivage prophasique; c'est à partir de la périphérie que cet élément est transformé.

Nous le savons déjà, puisque nous avons pris le noyau au repos comme point de départ de cette étude, le résultat de la télophase est la reconstitution d'un réseau chromatique constitué par un ensemble de granules petits et gros. Cette reconstitution s'opère de la façon suivante. Le chromosome est attaqué, comme érodé, par l'extérieur. Cette érosion, par places, ne désagrége pas le chromosome jusqu'à l'extrême limite, mais s'arrête au stade de gros grains. Par contre, dans d'autres portions, le chromosome est aminci au maximum et les minces filaments se fragmentent finalement en un grand nombre de très petits granules auxquels sont mélangés les chromocentres. On voit done ici s'accentuer la désagrégation du chromosome qui, par places, ne va pas jusqu'au stade ultime des granulations ténues, mais dépasse, par endroits, le stade de chromocentres auquel elle s'arrête dans le cas précédent.

$4^{\circ}$ ) TYPE Allium. - La fragmentation du chromosome est parfaite, pourrait-on dire, chez l'Allium ou l'Hyacinthus, par exemple. Là, en effet, on assiste d'abord à un amincissement considérable de l'élément chromosomique; on obtient alors des filaments ténus qui ultérieurement sont fragmentés en de très nombreux et très fins granules dont l'ensemble constitue un réseau régulier et finement granuleux dans toute son étendue.

Par tout ce qui précède, on voit que la fragmentation, à la télophase, des chromosomes est différemment prononcée suivant les cas. La gradation dans l'intensité du phénomène est la suivante: chez les noyaux à prochromosomes, aucune transformation à la télophase de l'élément chromosomique, c'est le cas le plus simple et comme le point de départ et le témoin des modifications qui se produisent ailleurs. Premier degré de désagrégation dans le cas des noyaux à chromocentres sans réseau, oú le chromosome est simplement scindé en un certain nombre d'éléments plus petits. Second degré de désagrégation, plus prononcé que le précédent, réalisé chez les végétaux à noyaux réticulés 
avec chromocentres. La pulvérisation du chromosome n'est pas absolue et, par places, des fragments entiers, ou chromocentres, sont respectés. Troisième degré de désagrégation qui conduit à la formation de très fins granules exclusivement, chez les noyaux à réseau uniforme. La fragmentation du chromosome se poursuit jusqu'à l'obtention de granulations toutes semblables et des plus petites.

Si l'on ne craignait de risquer une comparaison qui pourrait être qualifiée de romanesque, on serait tenté de rapprocher ces phénomènes de l'action mécanique de la mer sur les rochers qu'elle désagrège jusqu'à les transformer en galets et même en sable fin. Quant à connaître le mécanisme de cette pulvérisation du chromosome, plus ou moins accentuée, il n'y faut pas songer pour le moment, tout au plus pourrait-on en chercher la cause dans une action du suc nucléaire, constituant au sujet duquel nos connaissances sont réduites à peu près à rien.

\section{Bibliographie}

Eichhorn (A.). 1) Recherches caryologiques comparées chez les Angiospermes et les Gymnospermes, Arch. de Bot., v, Mém., n 1, 1-100, 4 pl., 1931.

2) La mitose somatique du Cotonnier, C.R. Soc. Biol., XCII, 260, 1933.

3) Sur l'existence de prochromosomes dans les noyaux de Sinapis nigra, C. $R$. Soc. Biol., CXII, 535, 1933.

4) Observation vitales sur les noyaux à structure non réticulée, C. $R$. Soc. Biol., CXII, 1625, 1933.

Eichhorn (A.) et Franquet (R.). 1) La mitose somatique des Cucurbitacées. Contribution à l'étude des noyaux porteurs de prochromosomes, Arch. du Muséum, VII, 205-224, 27 fig., 1931.

2) La mitose somatique chez le Fatshedera Lizei, C. R. Soc. Biol., CXII, 744, 1933.

Heitz (E.). Heterochromatin, Chromocentren, Chromomeren, Ber. d. d. bot. Gesell., XLVII, 274-284, 1 pl., 1929.

Kuhn (E.). Die Beziehung der Chromocentren zur Chromosomenbildung. Ber. $d$. d. bot. Gesell., XLVII, 420-430, 1929.

Litardière (R. de). Recherches sur l'élément chromosomique dans la caryocinèse somatique des Filicinées. La Cellule, XXXI, 255-475, 1921.

Nardi (R.). Structure et évolution des noyaux dans les propagules de Lunulara cruciata (L.), Dum. C. R. Soc. Biol., CXIII, 380, 1933.

Schiller (J.). UUber den Verlauf der Kernteilung bei Capparis mit Dauerchromosomen. Jahrb.f. wiss. Bot., LXIV, 491-500, 1928.

Sharp (L. W.). Introduction to Cytology. 1926.

Tischler (G.). Allgemeine Pflanzenkaryologie, in K. Linsbauer: Handbuch der Fflanzenanatomie, 1 Abt., 1 Teil, II Bd., Berlin, Borntraeger, 1921.

Laboratoire de Botanique, P. C. N. Paris. 